\title{
STATISTICAL ORBIT DETERMINATION ALGORITHM FOR SATELLITES IN INDIAN NAVIGATION CONSTELLATION (NavIC): TOWARDS EXTENDED EPHEMERIS TECHNOLOGY FOR NavIC RECEIVER
}

\author{
T. V. RAMANATHAN ${ }^{1}$, RADHIKA A. CHIPADE ${ }^{1,2}$ \\ ${ }^{1}$ Department of Statistics \& Centre for Advanced Studies \\ Savitribai Phule Pune University, Pune 411 007, India \\ ${ }^{2}$ Space Applications Centre (ISRO), Ahmedabad 380015, India \\ e-mails: ram@unipune.ac.in; radhikachipade@gmail.com
}

\begin{abstract}
Ephemerides are essential for the satellite positioning in Global Navigation Satellite Systems (GNSS) user receivers. Acquisition of navigation data and ephemeris parameters are difficult in remote areas as well as in challenging environments. Statistical orbit determination techniques can help to predict the orbital parameters in the absence of navigation data. The present study is a first step towards the solution for generating orbital parameters and predicting the satellite positions in the absence of navigation data for satellites in NavIC constellation. The orbit determination algorithm predicted the satellite position using single station navigation data. The perturbations affecting the satellite orbits in NavIC constellation were also studied and an algorithm using perturbation force models is proposed for the satellites in NavIC constellation. Extended Kalman Filter (EKF) was used to address the non-linear dynamics model of the perturbation forces and distance of the ground station from the centre of Earth was used as measurement to solve the measurement equation. The satellite orbits were predicted up to 1 hour using the single station navigation data. The root mean square error (RMSE) of $12.59 \mathrm{~m}$ and $13.03 \mathrm{~m}$ were observed for NavIC satellites in Geosynchronous and Geostationary orbits, respectively, after 1 hour. The Kolmogorov-Smirnov test used to assess the goodness of fit of the proposed EKF algorithm for orbit prediction was found to be significant at $1 \%$ level of significance.
\end{abstract}

Keywords: Extended Kalman Filter, NavIC, orbital parameters, statistical orbit determination

\section{INTRODUCTION}

Broadcast ephemeris is essential to determine the accurate position fix in navigation. However, GNSS receivers find it difficult to acquire the broadcast ephemeris from satellites in challenging environments such as inside a building, areas with dense plantation and so on. Hence, it becomes difficult to determine the position fix at the receiver. One way to overcome this difficulty is to generate satellite ephemeris data locally at the receiver instead of broadcast ephemeris. This is achieved by statistical orbit determination techniques locally at the receiver. Orbit determination is the process of estimating the state of a satellite, whose initial state is usually the subject of improving, based on the observations that are influenced by random and systematic

(C) The Author(s). This is an open-access article distributed under the terms of the Creative Commons Attribution License (CC BY 4.0, 
errors (Tapley et al., 2004). In orbit determination procedure, predicting the state of the satellite is called as "generating an ephemeris". Orbit determination algorithm will provide orbital parameters locally at the receiver and position fix can be achieved reducing the time to first fix (TTFF) of the receiver. If the receiver has a backup of valid ephemeris data stored, it will calculate its position after powering on within few seconds (hot start). However, in the absence of valid back up of the ephemeris data, the statistical orbit determination algorithms become an essential part of the extended ephemeris technology of the receiver.

Many researchers have reported different statistical orbit determination algorithms. Hein (1997) has reported a simulation study for geosynchronous satellites of a European Satellite Navigation System (ENSS) that uses Extended Kalman Filter (EKF) for orbit determination. The data from 8 tracking stations all over the world was used for the orbit determination. Carolipio et al. (2002) developed the Kalman filter based orbit determination algorithm for geostationary satellites which serve as relays to transmit messages for space-based augmentation systems (SBAS). Stacey and Ziebart (2011) and Seppanen et al. (2011) have reported the Least-Squares based approach for orbit determination of GPS satellites. These studies demonstrate the technology for mobile devices. Aghav and Gangal (2014) have attempted comparison between Least-Squares and Kalman filter on the basis of a single data point for a satellite in low-earth orbit. Accuracy of kilometer $(\mathrm{km})$ level was achieved for the orbit determination, which needs to be improved for meter (m) level positioning accuracy. Shou (2014) reported a study on Unscented Kalman filter (UKF) for orbit prediction of satellites in low earth orbits (LEO). A comparison between EKF and UKF showed that UKF satisfied the required position accuracy. Kavitha et al. (2015) demonstrated Adaptive Kalman Filter for orbit determination using twoway code-division multiple access (CDMA) range stations' data from India. The data from four tracking stations across India for GSAT-10 and IRNSS-1A satellites was utilized to demonstrate the performance of algorithm. Chandrasekhar et al. (2015) demonstrated the orbit prediction algorithm for simulated orbits of NavIC (formerly IRNSS) 1D, 1E, 1F and $1 \mathrm{G}$ for validity period of maximum up to 1 hour. Zaminpardaz et al. (2017) have used the NavIC L5 observations for attitude determination. However, scanty literature is available on the statistical orbit determination techniques applied to Geostationary and Geosynchronous orbits with reference to NavIC constellation using observations from single receiver rather than from a network.

NavIC constellation consists of 4 Geosynchronous (GSO) and 3 Geostationary (GEO) satellites. 3 GEOs are located at $32.5^{\circ} \mathrm{E}, 83^{\circ} \mathrm{E}$ and $131^{\circ} \mathrm{E}$, whereas the 4 GSOs have their longitude crossings at $55^{0} \mathrm{E}$ and $111.75^{\circ} \mathrm{E}$, two in each plane (www.isac.gov.in/navigation/ irnss.jsp). This constellation is designed to provide positioning services to users in India as well as around the Indian boundary. Extended service area of NavIC lies between the primary service area and the area enclosed by the rectangle from latitude $30^{\circ} \mathrm{S}$ to $50^{\circ} \mathrm{N}$ and longitude $30^{\circ} \mathrm{E}$ to $130^{\circ} \mathrm{E}$ (www.isro.gov. in/irnss-programme). International GNSS Service (IGS) has its stations all over the world and one of them is BSHM, located at Haifa, Israel $\left(32^{0} 46^{\prime} \mathrm{N}, 35^{0} 01^{\prime} \mathrm{E}\right)$. These IGS stations regularly upload the data to IGS data repository. An initiative was taken to develop the orbit determination algorithms for satellites in NavIC constellation using single receiver observations collected at BSHM, Haifa.

Orbit of the GNSS satellites, in particular NavIC satellites, can be computed using kinematic, dynamic and statistical approaches. Kinematic approach is purely a geometric approach that considers the range and carrier phase measurements of the satellite with respect to ground (Svehla and Rothacher, 2004). This approach does not consider the dynamics of the motion of the satellite. Dynamic approach on the other hand is based on numerical integration of the equation of motion of the satellite (Svehla and Rothacher, 2004). Kinematic approach, being a 
geometric approach, is less precise as compared to the dynamic approach of orbit determination. Statistical orbit determination is the modern approach of orbit determination, which does not merely use the information about the motion of the satellite, but also the observation vector of the satellite from the ground and the minimization of the observational errors that are statistical in nature (Tapley, 2004). The present paper reports the first study of its kind to develop statistical orbit determination algorithms using the navigation data autonomously, which has its application in extended ephemeris technology for NavIC user receivers.

\section{METHODOLOGY}

An algorithm for statistical orbit determination was developed to estimate the position of the satellite at time $t_{k}$, provided the initial position was known at time $t_{0}$. This involves two important sub-steps, that is, the orbit prediction (propagation) and the orbit determination. The orbit prediction for NavIC satellites was addressed using a fixed step-size $4^{\text {th }}$ order Runge-Kutta numerical integration method by integrating the Kepler's force model considering the effects of other perturbation forces, that is, Earth's oblateness, solar and lunar attraction and solar radiation pressure. Extended Kalman Filter (EKF) was used as orbit determination algorithm. Details are given in the subsequent sections.

\subsection{Statistical Orbit Determination Algorithm}

Let the satellite position vector $\boldsymbol{r}=(x, y, z)^{T}$, velocity vector $\boldsymbol{v}=\left(v_{x}, v_{y}, v_{z}\right)^{T}$ and their respective magnitudes $r$ and $\nu$ be known in Earth-Centered-Inertial (ECI) reference frame at some initial time epoch $t_{0}$. Thus, the initial state vector used for the orbit prediction algorithm is $\boldsymbol{X}=\left(x, y, z, v_{x}, v_{y}, v_{z}\right)^{T}$. The initial state vector is computed in ECI reference frame using single ephemeris data that are available at initial epoch $t_{0}$ as downloaded from IGS data repository (https://cddis.nasa. gov).

The set of force models that determine the acceleration $\boldsymbol{a}$ of the satellite due to various effects is (Chandrasekhar et al., 2015)

$$
\boldsymbol{a}=-\frac{\mu \boldsymbol{r}}{r^{3}}+\boldsymbol{a}_{h a r}+\boldsymbol{a}_{\text {sun }}+\boldsymbol{a}_{\text {moon }}+\boldsymbol{a}_{\text {srp }},
$$

where, the Kepler's force model can be written as

$$
a=-\frac{\mu r}{r^{3}}
$$

with $\mu$ as Earth's gravitational constant $\left(3.986004418 * 10^{14}\right)\left(\mathrm{m}^{3} / \mathrm{s}^{2}\right)$, $\boldsymbol{a}_{\text {har }}$ is the acceleration due to Earth's oblateness, $\boldsymbol{a}_{\text {sun }}$ is the acceleration due to solar attraction, $\boldsymbol{a}_{\text {moon }}$ is the acceleration due to lunar attraction and $\boldsymbol{a}_{\text {srp }}$ is the acceleration due to solar radiation pressure (SRP).

The acceleration due to Earth's oblateness was studied by considering $J_{2}, J_{3}$ and $J_{4}$ zonal perturbations. The flattening coefficients in the system WGS-84 were considered as $J_{2}=$ 1082.63e $-6, J_{3}=-2.5321531 e-6$ and $J_{4}=-1.6109876 e-6$ (Chobotov, 2002).

The Cannonball type solar radiation pressure model was considered in this study, given by (3).

$$
\boldsymbol{a}_{\text {srp }}=\mu_{s} P C_{r} \frac{A}{m}(A U)^{2} \frac{\boldsymbol{e}_{\text {sun }}}{r_{\text {sun }}^{3}},
$$

where $\mu_{s}$ is the shadow factor; $A U$ is the astronomical unit $(149597870.691 \mathrm{~km}) ; P$ is the solar radiation pressure at distance of $1 A U$ from the sun $\left(4.56 e-6 \mathrm{~N} \cdot \mathrm{m}^{-2}\right) ; C_{r}$ is the reflectivity coefficient of the satellite; $A$ is the surface area of the satellite; $m$ is the mass of the satellite, $\boldsymbol{e}_{\text {sun }}$ 
is the unit vector from satellite to Sun and $r_{\text {sun }}$ is distance from satellite to Sun. Since there is not yet a detail model for the prediction of NavIC satellite orbits in autonomous mode, Cannonball model of solar radiation pressure was considered in the present study in this first attempt. These set of equations constitute the dynamics model of the statistical orbit determination algorithms.

The dynamics and the observation model (also known as the measurement model) for the statistical orbit determination problem in canonical form can be stated as (see Tapley et al., 2004; Mashiku et al., 2012; Mashiku, 2013).

$$
\begin{aligned}
& \dot{\boldsymbol{X}}(t)=\boldsymbol{F}(\boldsymbol{X}, t)+\boldsymbol{u}(t), \\
& \boldsymbol{Y}(t)=\boldsymbol{G}(\boldsymbol{X}, t)+\boldsymbol{w}(t),
\end{aligned}
$$

where $\boldsymbol{X}(t)$ is the state vector, $\boldsymbol{Y}(t)$ is a set of observations available as a function of the state vector $\boldsymbol{X}(t)$,

$$
\boldsymbol{F}(\boldsymbol{X}, t)=\left[\nu_{x}, \nu_{y}, \nu_{z},-\frac{\mu}{r^{3}} x,-\frac{\mu}{r^{3}} y,-\frac{\mu}{r^{3}} z\right]^{T},
$$

and $\boldsymbol{G}(\boldsymbol{X}, t)$ is a function of $\boldsymbol{X}(t)$ such that $\boldsymbol{G}(\boldsymbol{X}, t)$ is the vector $[r(t)]$ with

$$
r(t)=\sqrt{\left(x-x_{0}\right)^{2}+\left(y-y_{0}\right)^{2}+\left(z-z_{0}\right)^{2}},
$$

and $\left(x_{0}, y_{0}, z_{0}\right)$ are the coordinates of the receiver or the sensor on ground, which were assumed to be known. Note that $\boldsymbol{w}(t)$ is the observation (measurement) error (noise) with mean zero and a specified covariance matrix $\boldsymbol{R}$, and $\boldsymbol{u}(t)$ is the process noise with mean zero and a specified covariance matrix $\boldsymbol{Q}$.

The Extended Kalman filter (EKF) is nothing but a nonlinear counterpart of the Kalman filter and addresses the non-linear dynamics model of statistical orbit determination (Tapley et al., 2004). EKF was used to solve for the measurement model of the orbit determination algorithm. The following set of equations were used to estimate the state vector $\boldsymbol{X}(t)$. EKF also estimates the posterior covariance associated with the estimate $\hat{\boldsymbol{X}}_{k} ; \boldsymbol{P}\left(t_{k}\right)$ that quantifies the uncertainty of the state estimate, the details of which follow as in Mashiku (2013).

The algorithm was initialized with an initial position vector $\hat{\boldsymbol{X}}_{0}$ computed based on the previously recorded navigation data and an a priori covariance associated with state estimate, $\boldsymbol{P}_{0}$; for the dynamics model of orbit determination. At time $t_{k}\left(t_{0}+d t\right)$, the state vector and the error covariance was computed using the state transition matrix $\mathbf{A}$,

$$
\begin{aligned}
\hat{\boldsymbol{X}}_{k}^{-} & =\mathbf{A} \hat{\boldsymbol{X}}_{k-1} \\
\boldsymbol{P}_{k}^{-} & =\mathbf{A} \boldsymbol{P}_{k-1} \mathbf{A}^{T}+\mathbf{Q}
\end{aligned}
$$

where, the state transition matrix $\mathbf{A}$ is

$$
\mathbf{A}=I_{6 \times 6}+\frac{\partial \boldsymbol{F}}{\partial \boldsymbol{X}} d t=I_{6 \times 6}+\left[\begin{array}{cccccc}
0 & 0 & 0 & 1 & 0 & 0 \\
0 & 0 & 0 & 0 & 1 & 0 \\
0 & 0 & 0 & 0 & 0 & 1 \\
-\frac{\mu}{r^{3}}+\frac{3 \mu x^{2}}{r^{5}} & \frac{3 \mu x y}{r^{5}} & \frac{3 \mu x z}{r^{5}} & 0 & 0 & 0 \\
\frac{3 \mu x y}{r^{5}} & -\frac{\mu}{r^{3}}+\frac{3 \mu y^{2}}{r^{5}} & \frac{3 \mu y z}{r^{5}} & 0 & 0 & 0 \\
\frac{3 \mu x z}{r^{5}} & \frac{3 \mu y z}{r^{5}} & -\frac{\mu}{r^{3}}+\frac{3 \mu z^{2}}{r^{5}} & 0 & 0 & 0
\end{array}\right] d t .
$$


The Kalman gain was computed from the measurement models using the $\boldsymbol{H}$-matrix

$$
\begin{aligned}
\boldsymbol{K}_{k} & =\boldsymbol{P}_{k}^{-} \boldsymbol{H}^{T}\left(\boldsymbol{H} \boldsymbol{P}_{k}^{-} \boldsymbol{H}^{T}+\boldsymbol{R}\right)^{-1}, \\
\boldsymbol{H} & =\frac{\partial \boldsymbol{G}}{\partial \boldsymbol{X}} .
\end{aligned}
$$

In this study, distance of the satellite from the centre of Earth was considered as $r(t)$, to make the algorithm simplified and autonomous, independent of any receiver observable. Thus, $\left(x_{0}, y_{0}, z_{0}\right)$ was considered to be $(0,0,0)$.

The state estimate was updated using the Kalman gain and the innovation term was defined by $\left(\boldsymbol{Y}_{k}-\boldsymbol{H} \hat{\boldsymbol{X}}_{k}^{-}\right)$, where $\boldsymbol{Y}_{k}$ is the observation vector and

$$
\begin{aligned}
\hat{\boldsymbol{X}}_{k} & =\hat{\boldsymbol{X}}_{k}^{-}+\boldsymbol{K}_{k}\left(\boldsymbol{Y}_{k}-\boldsymbol{H} \hat{\boldsymbol{X}}_{k}^{-}\right), \\
\boldsymbol{P}_{k} & =\left(\boldsymbol{I}-\boldsymbol{K}_{k} \boldsymbol{H}\right) \boldsymbol{P}_{k}^{-} .
\end{aligned}
$$

The time step of 1 second ( $d t=1 \mathrm{sec}$.) was used to estimate the state $\boldsymbol{X}(t)$. The estimated state vector was in ECI reference frame and was further converted to radial, along track and cross track (RSW) reference frame using equation (11) given below:

$$
\hat{\boldsymbol{X}}_{k}^{R S W}=\left[\begin{array}{ccc}
\cos u & -\sin u & 0 \\
\sin u & \cos u & 0 \\
0 & 0 & 1
\end{array}\right]\left[\begin{array}{ccc}
1 & 0 & 0 \\
0 & \cos i & \sin i \\
0 & -\sin i & \cos i
\end{array}\right]\left[\begin{array}{ccc}
\cos \Omega & -\sin \Omega & 0 \\
-\sin \Omega & \cos \Omega & 0 \\
0 & 0 & 1
\end{array}\right] \hat{\boldsymbol{X}}_{k}^{E C I},
$$

where $u$ is the argument of latitude, $i$ is the angle of inclination and $\Omega$ is the right ascension of ascending node (RAAN) or longitude of ascending node (Vallado and McClain, 2007; Beutler and Mervart, 2010).

The satellite coordinates in radial direction were used to evaluate the accuracy of the predicted state vector of satellite position as the ranging errors primarily come from the ranging component of the orbit error (Misra and Enge, 2001).

\subsection{Criterion Used for Evaluation of Predicted Orbit Accuracy}

Root mean square error (RMSE) was used in the present study to evaluate the orbit accuracy. The predicted satellite coordinates using the statistical orbit determination algorithm were compared with the satellite coordinates computed using live recorded navigation data at station BSHM using equation (12)

$$
R M S E=\sqrt{\frac{\sum_{i=1}^{T}\left(Q_{i, p r e d}-Q_{i, a c t}\right)^{2}}{T}},
$$

where, $T$ is the total number of samples in the assumed interval over which the orbit was predicted. The value of $T$ varied depending on the availability of navigation data in the data-log file for a particular satellite. $Q_{\text {pred }}$ and $Q_{a c t}$ are predicted and estimated satellite coordinates in radial direction, respectively.

The Kolmogorov-Smirnov (K-S) test is a non-parametric test used for comparing two distributions, by quantifying the distance between the empirical cumulative distribution functions of two samples. K-S test was used to assess the goodness of fit of the orbit prediction algorithm by analyzing the distribution of actual and predicted satellite coordinates in the radial direction. 
The state vector of satellite position was estimated using only Kepler's model and Kepler's model with other perturbation forces. The decision regarding the choice of the force model to be used for orbit prediction was taken based on the RMSE errors in radial direction and the results of the K-S goodness of fit test. Figure 1 shows the flowchart of the developed algorithm.

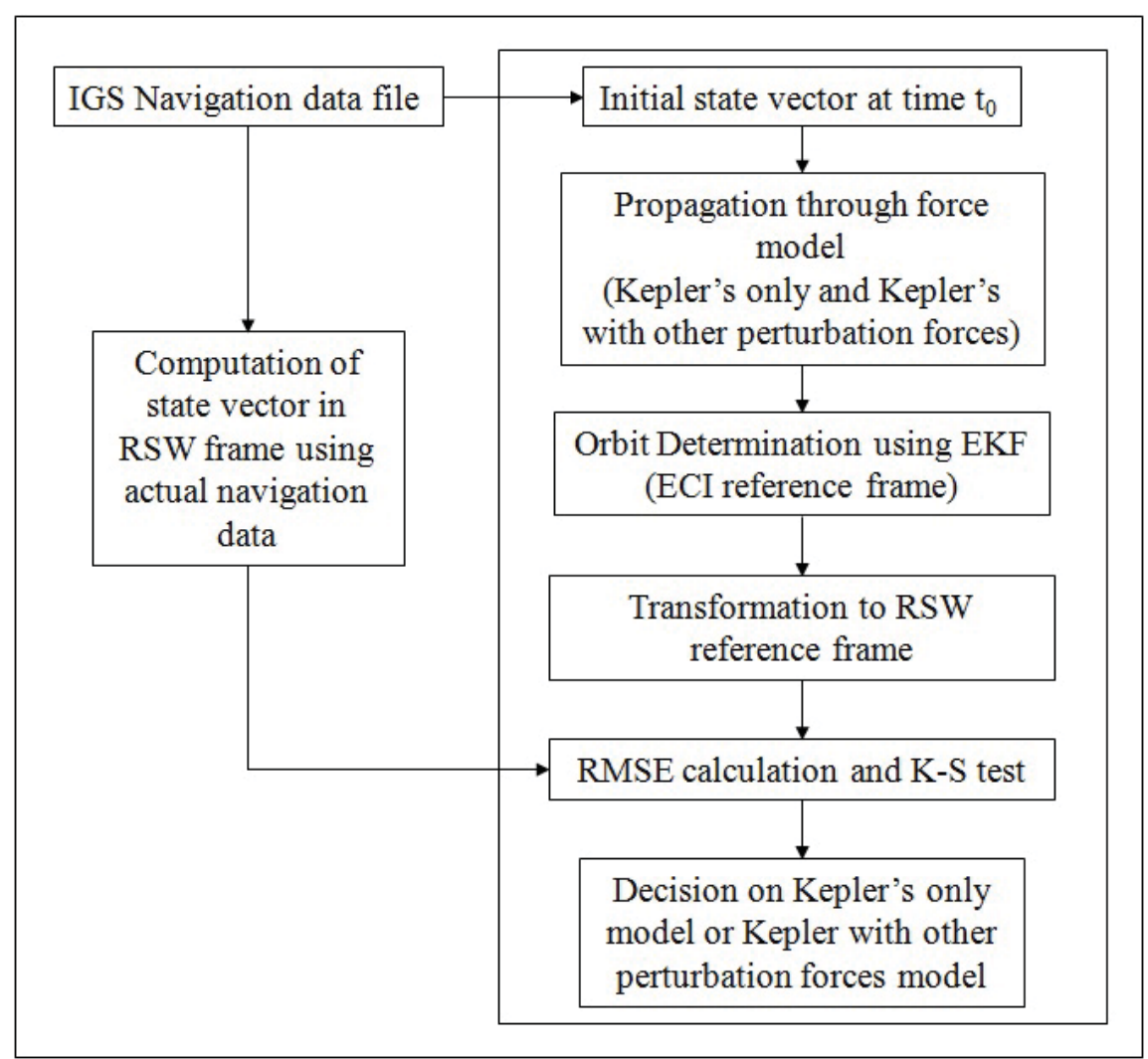

Figure 1. Flowchart of the algorithm developed for the statistical orbit determination

\subsection{Estimation of Orbital Parameters from the State Vector}

The estimated satellite position and velocity were further used to estimate the orbital parameters. The semi major axis, eccentricity, inclination angle, argument of perigee, longitude of ascending node and true anomaly were estimated using the estimated state vector of satellite as follows (Vallado and McClain, 2007; Beutler and Mervart, 2010).

\section{(a) Semi-Major Axis:}

Semi-major axis of an orbit can be calculated as

$$
a=\frac{\mu}{\left((2 \mu / r)-v^{2}\right)}
$$

where, $r$ and $v$ are magnitude of position and velocity vector, respectively.

\section{(b) Eccentricity Vector:}

Eccentricity vector can be computed as

$$
\boldsymbol{e}=\frac{1}{\mu}\left[\left(v^{2}-\frac{\mu}{r}\right) \boldsymbol{r}-(\boldsymbol{r} \cdot \boldsymbol{v}) \boldsymbol{v}\right] .
$$

The norm of eccentricity vector is the eccentricity of the satellite orbit. 


\section{(c) Angle of Inclination:}

Angle of inclination is calculated as

$$
i=\cos ^{-1}\left(\frac{\boldsymbol{k} \cdot \boldsymbol{l}}{\|\boldsymbol{l}\|}\right)
$$

where, $\boldsymbol{k}$ is unit vector pointing towards north pole and $\boldsymbol{l}=\boldsymbol{r} \times \boldsymbol{v}$ is angular momentum.

\section{(d) Argument of Perigee:}

Argument of perigee is calculated as

$$
\omega=\cos ^{-1}\left(\frac{\boldsymbol{n} \cdot \boldsymbol{e}}{\|\boldsymbol{n}\|\|\boldsymbol{e}\|}\right)
$$

where, $\boldsymbol{n}$ is the node vector, defined by $\boldsymbol{n}=(\boldsymbol{k} \times \boldsymbol{l}) /\|\boldsymbol{l}\|$.

\section{(e) Longitude of Ascending Node:}

Longitude of ascending node is calculated as

$$
\Omega=\cos ^{-1}\left[\frac{n_{x}}{\|\boldsymbol{n}\|}\right]
$$

where, $n_{x}$ is the $x$-component of node vector.

\section{(f) True Anomaly:}

True anomaly is calculated as

$$
\nu=\cos ^{-1}\left(\frac{\boldsymbol{e} \cdot \boldsymbol{r}}{\|\boldsymbol{e}\|\|\boldsymbol{r}\|}\right) .
$$

These estimated orbital parameters were further validated by comparing with actual parameters in navigation data file.

\section{RESULTS AND DISCUSSION}

Coordinates for the satellites in the NavIC constellation were estimated using the Extended Kalman Filter based statistical orbit determination algorithm and the prediction accuracy was analyzed using statistical procedures. Navigation data for NavIC satellites tracked on June 29, 2019 at IGS station BSHM located at Haifa, Israel was used in the present study (https://cddis. nasa.gov). The station coordinates were estimated using on-line positioning user services at https://www.ngs.noaa.gov/OPUS/ and are shown in Table 1 in Earth-Centered-Earth-Fixed (ECEF) reference frame. It was observed that the navigation data were available at irregular time intervals for different satellites of NavIC, identified with different pseudo random noise codes (PRNs). Thus, initial value was taken at the start of the log file for two of the NavIC satellites, that is, PRN 2 and PRN 6. Satellite identified with PRN 2 is in GSO, whereas, that identified by PRN 6 is in GEO (http://mgex.igs.org/IGS_MGEX_Status_IRNS.php).

Table 1. BSHM station coordinates in ECEF reference frame (in $\mathrm{m}$ )

\begin{tabular}{|c|c|c|c|}
\hline ECEF X & ECEF Y & ECEF Z & Precision \\
\hline 4395951.170 & 3080707.235 & 3433498.287 & 0.0256 \\
\hline
\end{tabular}


EKF algorithm was initialized with the satellite coordinates in ECI reference frame computed at time $t_{0}$. Table 2 shows the initial position vector at $t_{0}$ for both the NavIC satellites in ECI reference frame. The forces due to external bodies like sun and moon, radiation pressure affects the motion of satellites in space significantly. The amount of these forces for NavIC satellites is as shown in Table 3. Thus, these effects were considered while predicting the satellite orbits.

Table 2. Initial position vector for NavIC satellites tracked at BSHM (in m)

\begin{tabular}{|c|c|c|c|}
\hline Satellite & ECI X & ECI Y & ECI Z \\
\hline PRN 2 & 20490629.785 & 33339602.188 & 15867402.796 \\
\hline PRN 6 & 32628391.947 & 26707328.963 & 1862922.888 \\
\hline
\end{tabular}

Table 3. Acceleration due to different bodies affecting NavIC satellites

\begin{tabular}{|c|c|c|c|c|}
\hline Factor & $\begin{array}{c}\text { Earth's } \\
\text { Oblateness }\end{array}$ & $\begin{array}{c}\text { Solar } \\
\text { Attraction }\end{array}$ & $\begin{array}{c}\text { Lunar } \\
\text { Attraction }\end{array}$ & $\begin{array}{c}\text { Solar Radiation } \\
\text { Pressure }\end{array}$ \\
\hline Acceleration $\left(\mathrm{m} / \mathrm{s}^{2}\right)$ & $8.2823 \mathrm{e}-6$ & $2.1054 \mathrm{e}-6$ & $8.3247 \mathrm{e}-6$ & $1.5405 \mathrm{e}-8$ \\
\hline
\end{tabular}

The simplification of the navigation satellite to the cannonball model may appear to be insufficient in terms of the non-gravitational perturbing forces, such as the direct solar radiation pressure. Although the NavIC satellites have smaller boxes as compared to the other satellites, (e.g., GPS, Galileo, GLONASS, or QZSS), the acceleration resulting from the direct SRP is questionable, as it comprises about $10-15 \%\left(15 \mathrm{~nm} / \mathrm{s}^{2}\right.$ - Table 3$)$ of the accelerations acting on the GPS or Galileo satellites, for which the magnitude of SRP exceed $100 \mathrm{~nm} / \mathrm{s}^{2}$. However, it may be mentioned that there is no in-house model developed for NavIC constellation. This paper being the first attempt of its kind to predict the satellite orbits for NavIC satellites, the SRP model used by GPS and Galileo was used for developing the algorithm (in this context, see Hein and Eissfeller (1997), Seppanen et al. (2011) and Stacey and Ziebart (2011)). Further, the study was carried out with the aim to implement this algorithm in the receiver, which can be used in challenging environments. Thus, there is a trade-off between model complexity and the time taken for processing, which must be maintained by considering the degradation in the position error (see Zhang et al., 2008). Thus, we have decided to use the Cannonball model for orbit determination. Due to NavIC satellite size, weight and its GEO/GSO orbits, the solar radiation pressure value was observed to be low.

The diagonal elements of a priori covariance matrix $\left(\boldsymbol{P}_{0}\right)$ associated with state vector were initialized as $(10 \mathrm{~m}, 10 \mathrm{~m}, 10 \mathrm{~m}, 0.1 \mathrm{~m} / \mathrm{s}, 0.01 \mathrm{~m} / \mathrm{s}, 0.01 \mathrm{~m} / \mathrm{s})$. Unit Measurement covariance $(\mathbf{R})$ was considered, whereas, the diagonal elements of process noise covariance $(\mathbf{Q})$ were initialized at $(0.001 \mathrm{~m}, 0.001 \mathrm{~m}, 0.001 \mathrm{~m}, 1 \mathrm{e}-6 \mathrm{~m} / \mathrm{s}, 1 \mathrm{e}-6 \mathrm{~m} / \mathrm{s}, 1 \mathrm{e}-6 \mathrm{~m} / \mathrm{s})$.

The satellite orbits were predicted up to $1 \mathrm{hr}$ due to irregular time intervals between the two consecutive sets of ephemerides in the navigation file. Table 4 shows the errors in the radial component of the satellite orbit at every 30 minutes when only Kepler's force model and Kepler's force model with other perturbation forces were considered. Table 4 shows that RMSE increased when all the perturbation effects were considered for propagating the satellite orbit of GSO satellite, whereas for GEO, the values of RMSE were observed to be comparable for Kepler's model and Perturbation force model. This is due to oscillatory variation in the residual error of the radial component over period of time. In this study the prediction up to 1 hour was considered due to irregular time intervals, and thus, the oscillatory variation in residual error showed more RMSE in case of Perturbation force model. Magnitude of error in prediction was 
observed to be more for GEO satellite as compared to GSO satellite. Table 5 shows the RMSE values in the radial component on prediction through dynamic orbit determination. The increase in RMSE on prediction through dynamic orbit determination was observed not to be significant over EKF model for prediction up to 1 hour. This indicates that prediction through EKF was as good as that through dynamic model up to 1 hour.

Table 4. RMSE in the radial component on prediction through EKF

\begin{tabular}{|c|c|c|c|c|}
\hline Model $\rightarrow$ & \multicolumn{2}{|c|}{ Kepler's Force Model } & \multicolumn{2}{|c|}{$\begin{array}{r}\text { Kepler's + Perturbations } \\
\text { Force Model }\end{array}$} \\
\hline $\begin{array}{c}\text { NavIC } \\
\text { Satellites } \downarrow\end{array}$ & \multicolumn{2}{|c|}{$\begin{array}{c}\text { RMSE in Radial } \\
\text { Component (in m) }\end{array}$} & \multicolumn{2}{|c|}{$\begin{array}{r}\text { RMSE in Radial } \\
\text { Component (in m) }\end{array}$} \\
\hline & 30 min. & $1 \mathrm{hr}$. & 30 min. & $1 \mathrm{hr}$. \\
\hline PRN 2 & 9.63 & 27.83 & 12.59 & 39.33 \\
\hline PRN 6 & 13.30 & 42.67 & 13.03 & 42.69 \\
\hline
\end{tabular}

Table 5. RMSE in the radial component on prediction through Dynamic Orbit Determination

\begin{tabular}{|c|c|c|c|c|}
\hline Model $\rightarrow$ & \multicolumn{2}{|c|}{ Kepler's Force Model } & \multicolumn{2}{|c|}{$\begin{array}{c}\text { Kepler's + Perturbations } \\
\text { Force Model }\end{array}$} \\
\hline $\begin{array}{c}\text { NavIC } \\
\text { Satellites } \downarrow\end{array}$ & \multicolumn{2}{|c|}{$\begin{array}{c}\text { RMSE in Radial } \\
\text { Component (in m) }\end{array}$} & \multicolumn{2}{|c|}{$\begin{array}{c}\text { RMSE in Radial } \\
\text { Component (in m) }\end{array}$} \\
\hline & 30 min. & $1 \mathrm{hr}$. & 30 min. & $1 \mathrm{hr}$. \\
\hline PRN 2 & 9.71 & 27.92 & 12.68 & 39.43 \\
\hline PRN 6 & 13.38 & 42.76 & 13.11 & 42.78 \\
\hline
\end{tabular}

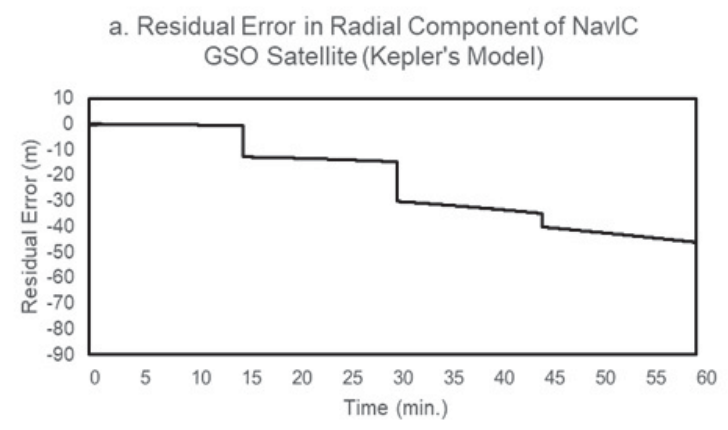

b. Residual Error in Radial Component of NavlC GSO Satellite (Perturbation Force Model)

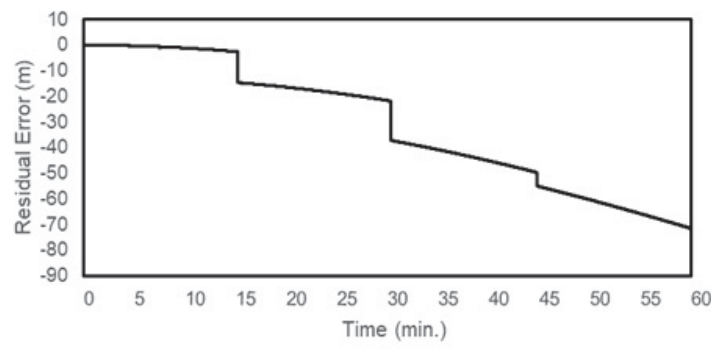

c. Residual Error in Radial Component of NaviC GEO Satellite (Kepler's Model)
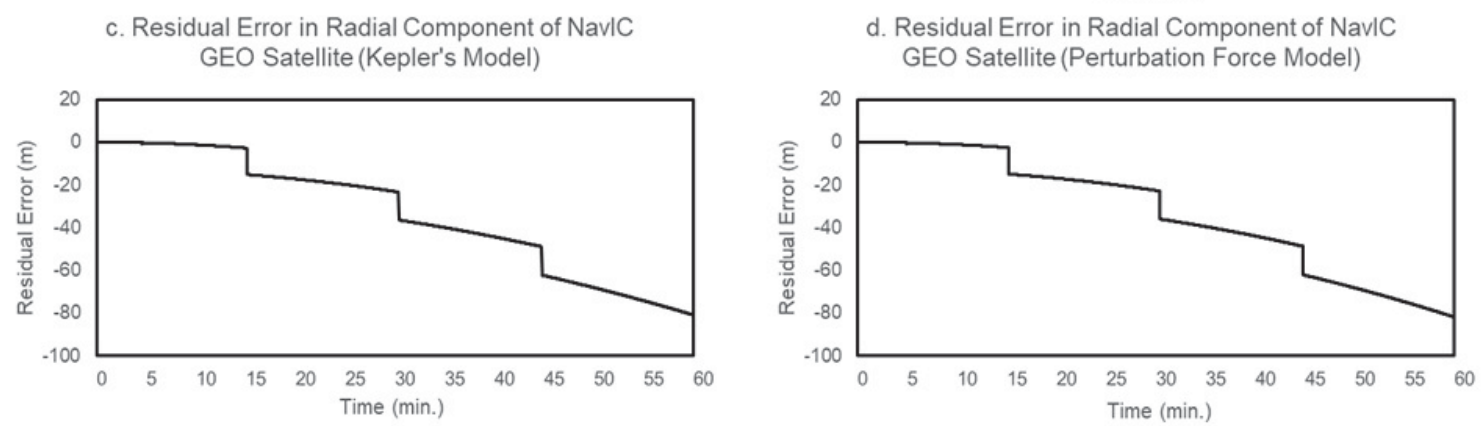

Figure 2. Residual Error in the radial component of NavIC

(a) GSO (Kepler's Model) (b) GSO (Perturbation Force Model) (c) GEO (Kepler's Model) and (d) GEO (Perturbation Force Model) satellites 
Figure 2 shows the variation in the residual error in the radial component of the NavIC satellites. In the case of NavIC satellites, ephemerides are uploaded at every 2 hours from the ground station. However, it was observed that new set of ephemerides were available at irregular time intervals of shorter than 1 hour leading to step changes in the residual error in the radial component of NavIC satellites (Figure 2).

Orbital parameters were also retrieved using the predicted satellite coordinates. Table 6 shows the RMSE in each of the retrieved orbital parameters given in Section 2.3, except the true anomaly as its actual value is not the part of set of ephemerides.

Table 6. RMSE in orbital parameters estimated using predicted orbit

\begin{tabular}{|c|c|c|c|c|c|c|}
\hline Satellite & Model & $\begin{array}{c}\text { sqrt(a) } \\
\left(\mathrm{m}^{1 / 2}\right)\end{array}$ & $\begin{array}{c}\text { Eccentricity } \\
(\mathrm{e})\end{array}$ & $\begin{array}{c}\text { Inclination } \\
(\mathrm{i} \text { in rad. })\end{array}$ & $\begin{array}{c}\text { RAAN } \\
(\Omega \text { in rad. })\end{array}$ & $\begin{array}{c}\text { Arg. of } \\
\text { Perigee } \\
(\omega \text { in rad. })\end{array}$ \\
\hline PRN 2 & Kepler's & 0.0577 & 0.0002 & $7.92 \mathrm{e}-6$ & $5.37 \mathrm{e}-5$ & 3.8226 \\
\hline PRN 6 & Kepler's & 0.1683 & 0.0002 & 0.4648 & 0.7508 & 0.0750 \\
\hline PRN 2 & $\begin{array}{c}\text { Kepler +Pertur- } \\
\text { bation forces }\end{array}$ & 0.0708 & 0.0002 & $5.54 \mathrm{e}-6$ & $1.44 \mathrm{e}-5$ & 2.8092 \\
\hline PRN 6 & $\begin{array}{c}\text { Kepler + Pertur- } \\
\text { bation forces }\end{array}$ & 0.2174 & 0.0002 & 0.4648 & 0.7502 & 0.0761 \\
\hline
\end{tabular}

Table 7. Test statistic and p-values of the Kolmogorov-Smirnov test in radial direction

\begin{tabular}{|c|c|c|c|c|c|}
\hline Satellite & Model & $\begin{array}{c}\text { Test } \\
\text { Statistic }\end{array}$ & p-value & $\begin{array}{c}\text { Decision at 5\% level } \\
\text { of significance }\end{array}$ & $\begin{array}{c}\text { Decision at 1\% level } \\
\text { of significance }\end{array}$ \\
\hline PRN 2 & Kepler's & 0.0071 & 0.5749 & $\begin{array}{c}\text { Hypothesis is } \\
\text { not rejected }\end{array}$ & $\begin{array}{c}\text { Hypothesis is } \\
\text { not rejected }\end{array}$ \\
\hline PRN 6 & Kepler's & 0.0195 & 0.0003 & $\begin{array}{c}\text { Hypothesis is } \\
\text { rejected }\end{array}$ & $\begin{array}{c}\text { Hypothesis is } \\
\text { rejected }\end{array}$ \\
\hline PRN 2 & $\begin{array}{c}\text { Kepler + Pertur- } \\
\text { bation forces }\end{array}$ & 0.0086 & 0.3283 & $\begin{array}{c}\text { Hypothesis is } \\
\text { not rejected }\end{array}$ & $\begin{array}{c}\text { Hypothesis is } \\
\text { not rejected }\end{array}$ \\
\hline PRN 6 & $\begin{array}{c}\text { Kepler + Pertur- } \\
\text { bation forces }\end{array}$ & 0.0059 & 0.8029 & $\begin{array}{c}\text { Hypothesis is } \\
\text { not rejected }\end{array}$ & $\begin{array}{c}\text { Hypothesis is } \\
\text { not rejected }\end{array}$ \\
\hline
\end{tabular}

Kolmogorov-Smirnov (K-S) test was performed to assess the goodness of fit of the EKF algorithm used for orbit prediction. Table 7 shows the test-statistic values and p-values for the K-S test performed on the radial component of satellite orbit as it is the primary factor responsible for ranging errors. The hypothesis that there is no significant difference in the distributions of actual and predicted radial components of the satellites was tested using K-S test. The decision on the hypothesis of the K-S test for the radial component of satellite orbit is given in Table 7. At $5 \%$ level of significance, it was observed that the actual values and predicted values of the radial component of the satellite orbit come from the same distribution, except for PRN 6 when only Kepler's model was used for propagation. At $1 \%$ level of significance, significant difference in the distributions of the actual values and predicted values of the randial component of the satellite orbit was observed when Kepler's model was used for orbit propagation for GEO satellite (PRN 6) of NavIC. Table 6 also shows that error values were less in the estimated orbital parameters when perturbation forces were considered, except the semi-major axis for a satellite in GEO. RMSE up to $13.03 \mathrm{~m}$ was achieved in the prediction of orbit after 30 minutes at time step of 1 second. These results thus show that the perturbation force model is the appropriate choice for orbit prediction where the K-S test was observed to be significant at both, 
$5 \%$ and $1 \%$ level of significance. This paper being the first attempt to predict the satellite orbit of NavIC using single station data, the accuracy comparison with the results of other research groups is not possible at this juncture.

\section{CONCLUSIONS}

A statistical orbit determination algorithm has been developed to predict the satellite coordinates in ECI reference frame using EKF and through perturbation force model. Simplified measurement model using position of the satellite from the centre of Earth was proposed to retrieve the satellite orbit autonomously. Meter level prediction accuracy was achieved in estimating the satellite coordinates using the proposed algorithm when validating the algorithm using the NavIC receiver data logged at IGS station. Kolmogorov-Smirnov test established the goodness of fit of the estimated orbit using the proposed algorithm. This is the first step towards addressing the extended ephemeris technology development for NavIC user receiver. This study can be further expanded to retrieve satellite coordinates in ECEF reference frame for positioning application using NavIC user receiver in challenging environments. The perturbation force models used in this study add the complexity to the statistical orbit determination algorithm in terms of computation involved and time taken to predict the satellite coordinates. Thus, the study can be further expanded to have its real time application in self-assisted ephemeris technology for NavIC user receiver.

\section{Acknowledgements.}

This work is carried out in collaboration with the Space Applications Centre (ISRO) under the Ph.D. program. The authors are thankful to Mr. D. K. Das, Director, Space Applications Centre (SAC), Ahmedabad, for providing the opportunity to carry out this work. The authors are very grateful to Mr. Tapan Misra, Mr. N. M. Desai and Mr. A. P. Shukla, SAC/ISRO for their constant support towards this work. The authors also extend their sincere gratitude to the anonymous referees for their critical comments and constructive suggestions.

\section{REFERENCES}

Aghav, S. and Gangal, S. (2014) Simplified orbit determination algorithm for low earth orbit satellites using space-borne GPS navigation sensor, Artificial Satellites, Vol. 49, No. 2, 81-99.

Beutler, G. and Mervart, L. (2010) Methods of Celestial Mechanics. Vol. 1: Physical, Mathematical, and Numerical Principles, Springer Verlag, Berlin.

Carolipio, E. M., Pandya, N. K., Grewal, M. S. (2002) GEO orbit determination via covariance analysis with a known clock error, Navigation, Vol. 48, No. 4, 255-260.

Chobotov, V. A. (2002) Orbital Mechanics, 3rd Ed., AIAA, Virginia.

Chandrasekhar, M. V., Rajarajan, D., Satyanarayana, G., Tirmal, N., Rathnakara, S. C., Ganeshan, A. S. (2015) Modernized IRNSS broadcast ephemeris parameters, Control Theory and Informatics, Vol. 5, No. 2, 1-9.

Hein, G. W., Eissfeller B. (1997) Orbit determination of geosynchronous satellites of a European satellite navigation system (ENSS), Proc. 12th Int. Sym. "Space Flight Dynamics", $59-64$.

Kavitha, S., Mula, P., Babu, R., Ratnakara, S. C., Ganeshan, A.S. (2015) Adaptive extended Kalman filter for orbit estimation of GEO satellites, Journal of Environment and Earth Science, Vol. 5, No.3, 1-10. 
Mashiku, A. K., Garrison, J. L., Carpenter, J. R. (2012) Statistical orbit determination using the particle filter for incorporating non-Gaussian uncertainties, AIAA Astrodynamics Specialist Conference, August 13-16, Minneapolis, Minnesota.

Mashiku, A. K. (2013) Information Measures for Statistical Orbit Determination, Ph. D. Dissertation, Purdue University, Indiana.

Misra P. and Enge P. (2001) Gloabal Positioning System, Signals, Measurements and Performance, Ganga-Jamuna Press, Lincoln, Massachusetts.

Seppanen, M., Perala, T., Piche, R. (2011) Autonomous satellite orbit prediction, Proceedings of the 2011 International Technical Meeting of The Institute of Navigation, 554-564.

Shou, H. (2014) Orbit propagation and determination of low earth orbit satellites, International Journal of Antennas and Propagation, Article ID 903026.

Stacey, P. and Ziebart, M. (2011) Long-term extended ephemeris prediction for mobile devices, ION-GNSS, 3235-3244.

Tapley, B., Schutz, B., Born, G. (2004) Statistical Orbit Determination, Elsevier, New York.

Vallado, D., McClain, W. (2007) Fundamentals of Astrodynamics and Applications, 3rd ed., Microcosm Press, Canada.

Zaminpardaz, S., Teunnissen, P. J. G., Nadarajah, N. (2017) Single-frequency L5 attitude determination from IRNSS/NavIC and GPS: a single and dual-system analysis, Journal of Geodesy, Vol. 91, 1415-1433.

Zhang, W., V. Vijay, Liu H., Phatak, M., Han, S. (2008) SiRF InstantFix II Technology, ION GNSS, Vol. 3, 1616-1623.

Received: 17-07-2019

Reviewed: 24-04-2020 and 11-05-2020

Accepted: 11-05-2020 